Journal of Physics A 42 (2009) 465102

\title{
Differential Equations with Fractional Derivative and Universal Map with Memory
}

\author{
Vasily E. Tarasov ${ }^{1,2}$ \\ 1) Courant Institute of Mathematical Sciences, New York University \\ 251 Mercer Street, New York, NY 10012, USA \\ 2) Skobeltsyn Institute of Nuclear Physics, \\ Moscow State University, Moscow 119991, Russia \\ E-mail: tarasov@theory.sinp.msu.ru
}

\begin{abstract}
Discrete maps with long-term memory are obtained from nonlinear differential equations with Riemann-Liouville and Caputo fractional derivatives. These maps are generalizations of the well-known universal map. The memory means that their present state is determined by all past states with special forms of weights. To obtain discrete map from fractional differential equations, we use the equivalence of the Cauchy-type problems and to the nonlinear Volterra integral equations of second kind. General forms of the universal maps with memory, which take into account general initial conditions, for the cases of the Riemann-Liouville and Caputo fractional derivatives, are suggested.
\end{abstract}

\section{Introduction}

A dynamical system consists of a set of possible states, together with a rule that determines the present state in terms of past states. If we require that the rule be deterministic, then we can define the present state uniquely from the past states. A discrete-time system without memory takes the current state as input and updates the situation by producing a new state as output. All physical classical models are described by differential or integro-differential equations, and 
the discrete-time systems can be considered as a simplified version of these equations. A discrete form of the time evolution equation is called the map. Maps are important because they encode the behavior of deterministic systems. The assumption of determinism is that the output of the map can be uniquely determined from the input. In general, the present state is uniquely determined by all past states, and we have a discrete map with memory. Discrete maps are used for the study of evolution problems, possibly as a substitute of differential equations [1, 2, 3]. They lead to a much simpler formalism, which is particularly useful in simulations. The universal discrete map is one of the most widely studied maps. In this paper, we consider discrete maps with memory that can be used to study solutions of fractional differential equations [4, 5, 6, 7].

The nonlinear dynamics can be considered in terms of discrete maps. It is a very important step in understanding the qualitative behavior of systems described by differential equations. The derivatives of non-integer orders are a generalization of the ordinary differentiation of integer order. Fractional differentiation with respect to time is characterized by long-term memory effects. The discrete maps with memory are considered in [8, 9, 10, 11, 12, 13, 14]. The interesting question is a connection of fractional differential equations and discrete maps with memory. It is important to derive discrete maps with memory from the equation of motion.

In Ref. [14], we prove that the discrete maps with memory can be derived from differential equations with fractional derivatives. The fractional generalization of the universal map was obtained [14] from a differential equation with Riemann-Liouville fractional derivatives. The Riemann-Liouville derivative has some notable disadvantages such as the hyper-singular improper integral, where the order of singularity is higher than the dimension, and nonzero of the fractional derivative of constants, which would entail that dissipation does not vanish for a system in equilibrium. The desire to formulate initial value problems for mechanical systems leads to the use of Caputo fractional derivatives rather than Riemann-Liouville fractional derivative.

It is possible to state that the Caputo fractional derivatives allows us to give more clear mechanical interpretation. At the same time, we cannot state that the Riemann-Liouville fractional derivative does not have a physical interpretation and that it shows unphysical behavior. Physical interpretations of the Riemann-Liouville fractional derivatives are more complicated 
than Caputo fractional derivatives. But the Riemann-Liouville fractional derivatives naturally appear for real physical systems in electrodynamics. We note that the dielectric susceptibility of a wide class of dielectric materials follows, over extended frequency ranges, a fractional power-law frequency dependence that is called the "universal" response [15, 16]. As was proved in [17, 18], the electromagnetic fields in such dielectric media are described by differential equations with Riemann-Liouville fractional time derivatives. These fractional equations for "universal" electromagnetic waves in dielectric media are common to a wide class of materials, regardless of the type of physical structure, chemical composition, or of the nature of the polarizing species. Therefore, we cannot state that Riemann-Liouville fractional time derivatives do not have a physical interpretation. The physical interpretation of these derivatives in electrodynamics is connected with the frequency dependence of the dielectric susceptibility. As a result, the discrete maps with memory that are connected with differential equations with Riemann-Liouville fractional derivatives are very important, and these derivatives naturally appear for real physical systems.

For computer simulation and physical application, it is very important to take into account the initial conditions for discrete maps with memory that are obtained from differential equations with Riemann-Liouville fractional time derivatives. In Ref. [14, these conditions are not obtained. In this paper, to take into account the initial condition, we use the equivalence of the differential equation with Riemann-Liouville and Caputo fractional derivatives and the Volterra integral equation. This approach is more general than the auxiliary variable method that is used in Ref. [14]. The proof of the result for Riemann-Liouville fractional derivatives is more complicated in comparison with the results for the Caputo fractional derivative. In this paper, we prove that the discrete maps with memory can be obtained from differential equations with the Caputo fractional derivative. The fractional generalization of the universal map is derived from a fractional differential equation with Caputo derivatives.

The universal maps with memory are obtained by using the equivalence of the fractional differential equation and the Volterra integral equation. We reduce the Cauchy-type problem for the differential equations with the Caputo and Riemann-Liouville fractional derivatives to nonlinear Volterra integral equations of second kind. The equivalence of this Cauchy type 
problem for the fractional equations with the Caputo derivative and the correspondent Volterra integral equation was proved by Kilbas and Marzan in [19, 20]. We also use that the Cauchytype problem for the differential equations with the Riemann-Liouville fractional derivative can be reduced to a Volterra integral equation. The equivalence of this Cauchy-type problem and the correspondent Volterra equation was proved by Kilbas, Bonilla, and Trujillo in [21, 22].

In Section 2, differential equations with integer derivative and universal maps without memory are considered to fix notations and provide convenient references. In Section 3, fractional differential equations with the Riemann-Liouville derivative and universal maps with memory are discussed. In Section 4, the difference between the Caputo and Riemann-Liouville fractional derivatives are discussed. In Section 5, fractional differential equations with the Caputo derivative and correspondent discrete maps with memory are considered. A fractional generalization of the universal map is obtained from kicked differential equations with the Caputo fractional derivative of order $1<\alpha \leq 2$. The usual universal map is a special case of the universal map with memory. Finally, a short conclusion is given in section 6 .

\section{Integer derivative and universal map without memory}

In this section, differential equations with derivative of integer order and the universal map without memory are considered to fix notations and provide convenient references.

Let us consider the equation of motion

$$
D_{t}^{2} x(t)+K G[x(t)] \sum_{k=1}^{\infty} \delta\left(\frac{t}{T}-k\right)=0
$$

in which perturbation is a periodic sequence of delta-function-type pulses (kicks) following with period $T=2 \pi / \nu, K$ is an amplitude of the pulses, $D_{t}^{2}=d^{2} / d t^{2}$, and $G[x]$ is some real-valued function. It is well-known that this differential equation can be represented in the form of the discrete map

$$
x_{n+1}-x_{n}=p_{n+1} T, \quad p_{n+1}-p_{n}=-K T G\left[x_{n}\right] .
$$

Equations (2) are called the universal map. For details, see for example [1, 2, 3]. 
The traditional method of derivation of the universal map equations from the differential equations is considered in Section 5.1 of [2]. We use another method of derivation of these equations to fix notations and provide convenient references. We obtain the universal map by using the equivalence of the differential equation and the Volterra integral equation.

Proposition 1. The Cauchy-type problem for the differential equations

$$
\begin{gathered}
D_{t}^{1} x(t)=p(t) \\
D_{t}^{1} p(t)=-K G[x(t)] \sum_{k=1}^{\infty} \delta\left(\frac{t}{T}-k\right),
\end{gathered}
$$

with the initial conditions

$$
x(0)=x_{0}, \quad p(0)=p_{0}
$$

is equivalent to the universal map equations of the form

$$
\begin{gathered}
x_{n+1}=x_{0}+p_{0}(n+1) T-K T^{2} \sum_{k=1}^{n} G\left[x_{k}\right](n+1-k), \\
p_{n+1}=p_{0}-K T \sum_{k=1}^{n} G\left[x_{k}\right] .
\end{gathered}
$$

Proof. Consider the nonlinear differential equation of second order

$$
D_{t}^{2} x(t)=G[t, x(t)], \quad\left(0 \leq t \leq t_{f}\right)
$$

on a finite interval $\left[0, t_{f}\right]$ of the real axis, with the initial conditions

$$
x(0)=x_{0}, \quad\left(D_{t}^{1} x\right)(0)=p_{0} .
$$

The Cauchy-type problem of the form (8), (9) is equivalent to the Volterra integral equation of second kind

$$
x(t)=x_{0}+p_{0} t+\int_{0}^{t} d \tau G[\tau, x(\tau)](t-\tau) .
$$

Using the function

$$
G[t, x(t)]=-K G[x(t)] \sum_{k=1}^{\infty} \delta\left(\frac{t}{T}-k\right),
$$


for $n T<t<(n+1) T$, we obtain

$$
x(t)=x_{0}+p_{0} t-K T \sum_{k=1}^{n} G[x(k T)](t-k T) .
$$

For the momentum $p(t)=D_{t}^{1} x(t)$, equation (11) gives

$$
p(t)=p_{0}-K T \sum_{k=1}^{n} G[x(k T)] .
$$

The solution of the left side of the $(n+1)$ th kick

$$
\begin{aligned}
& x_{n+1}=x\left(t_{n+1}-0\right)=\lim _{\varepsilon \rightarrow 0+} x(T(n+1)-\varepsilon), \\
& p_{n+1}=p\left(t_{n+1}-0\right)=\lim _{\varepsilon \rightarrow 0+} p(T(n+1)-\varepsilon),
\end{aligned}
$$

where $t_{n+1}=(n+1) T$, has the form (6) and (7) .

This ends the proof.

\section{Remark 1 .}

We note that equations (6) and (7) can be rewritten in the form (2). Using equations (6) and (17), the differences $x_{n+1}-x_{n}$ and $p_{n+1}-p_{n}$ give equations (2) of the universal map.

\section{Remark 2.}

If $G[x]=-x$, then equations (2) give the Anosov-type system

$$
x_{n+1}-x_{n}=p_{n+1} T, \quad p_{n+1}-p_{n}=K T x_{n} .
$$

For $G[x]=\sin (x)$, equations (2) are

$$
x_{n+1}-x_{n}=p_{n+1} T, \quad p_{n+1}-p_{n}=-K T \sin \left(x_{n}\right) .
$$

This map is known as the standard or Chirikov-Taylor map [1]. 


\section{Riemann-Liouville fractional derivative and universal map with memory}

In this section, we discuss nonlinear differential equations with the left-sided Riemann-Liouville fractional derivative ${ }_{0} D_{t}^{\alpha}$ defined for $\alpha>0$ by

$$
{ }_{0} D_{t}^{\alpha} x(t)=D_{t}^{n}{ }_{0} I_{t}^{n-\alpha} x(t)=\frac{1}{\Gamma(n-\alpha)} \frac{d^{n}}{d t^{n}} \int_{0}^{t} \frac{x(\tau) d \tau}{(t-\tau)^{\alpha-n+1}}, \quad(n-1<\alpha \leq n),
$$

where $D_{t}^{n}=d^{n} / d t^{n}$, and ${ }_{0} I_{t}^{\alpha}$ is a fractional integration [4, 7, 6].

We consider the fractional differential equation

$$
{ }_{0} D_{t}^{\alpha} x(t)=G[t, x(t)]
$$

where $G[t, x(t)]$ is a real-valued function, $0 \leq n-1<\alpha \leq n$, and $t>0$, with the intial conditions

$$
\left({ }_{0} D_{t}^{\alpha-k} x\right)(0+)=c_{k}, \quad k=1, \ldots, n .
$$

The notation $\left({ }_{0} D_{t}^{\alpha-k} x\right)(0+)$ means that the limit is taken at almost all points of the right-sided neighborhood $(0,0+\varepsilon), \varepsilon>0$, of zero as follows

$$
\begin{gathered}
\left({ }_{0} D_{t}^{\alpha-k} x\right)(0+)=\lim _{t \rightarrow 0+}{ }_{0} D_{t}^{\alpha-k} x(t), \quad(k=1, \ldots, n-1), \\
\left({ }_{0} D_{t}^{\alpha-n} x\right)(0+)=\lim _{t \rightarrow 0+}{ }_{0} I_{t}^{n-\alpha} x(t) .
\end{gathered}
$$

The Cauchy-type problem (18) and (19) can be reduced to the nonlinear Volterra integral equation of second kind

$$
x(t)=\sum_{k=1}^{n} \frac{c_{k}}{\Gamma(\alpha-k+1)} t^{\alpha-k}+\frac{1}{\Gamma(\alpha)} \int_{0}^{t} \frac{G[\tau, x(\tau)] d \tau}{(t-\tau)^{1-\alpha}}
$$

where $t>0$. The result was obtained by Kilbas, Bonilla, and Trujillo in [21, 22]. For $\alpha=n=2$, equation (20) gives (10).

The Cauchy-type problem (18) and (19) and the nonlinear Volterra integral equation (20) are equivalent in the sense that, if $x(t) \in L\left(0, t_{f}\right)$ satisfies one of these relations, then it also 
satisfies the other. In [21, 22] (see also Theorem 3.1. in Section 3.2.1 of [7]), this result is proved by assuming that the function $G[t, x]$ belongs to $L\left(0, t_{f}\right)$ for any $x \in W \subset \mathbb{R}$.

Let us give the basic theorem regarding the nonlinear differential equation involving the Riemann-Liouville fractional derivative.

Kilbas-Bonilla-Trujillo Theorem. Let $W$ be an open set in $\mathbb{R}$ and let $G[t, x]$, where $t \in\left(0, t_{f}\right]$ and $x \in W$, be a real-valued function such that $G[t, x] \in L\left(0, t_{f}\right)$ for any $x \in W$. Let $x(t)$ be a Lebesgue measurable function on $\left(0, t_{f}\right)$. If $x(t) \in L\left(0, t_{f}\right)$, then $x(t)$ satisfies almost everywhere equation (18) and conditions (19) if, and only if, $x(t)$ satisfies almost everywhere the integral equation (20).

Proof. This theorem is proved in [21, 22] (see also Theorem 3.1. in Section 3.2.1 of [7]).

In Ref. [14] we consider a fractional generalization of equation (11) of the form

$$
{ }_{0} D_{t}^{\alpha} x(t)+K G[x(t)] \sum_{k=1}^{\infty} \delta\left(\frac{t}{T}-k\right)=0, \quad(1<\alpha \leq 2),
$$

where $t>0$, and ${ }_{0} D_{t}^{\alpha}$ is the Riemann-Liouville fractional derivative defined by (17). Let us give the following theorem for equation (21).

Proposition 2. The Cauchy-type problem for the fractional differential equation of the form (21) with the initial conditions

$$
\left({ }_{0} D_{t}^{\alpha-1} x\right)(0+)=c_{1}, \quad\left({ }_{0} D_{t}^{\alpha-2} x\right)(0+)=\left({ }_{0} I_{t}^{2-\alpha} x\right)(0+)=c_{2}
$$

is equivalent to the equation

$$
x(t)=\frac{c_{1}}{\Gamma(\alpha)} t^{\alpha-1}+\frac{c_{2}}{\Gamma(\alpha-1)} t^{\alpha-2}-\frac{K T}{\Gamma(\alpha)} \sum_{k=1}^{n} G[x(k T)](t-k T)^{\alpha-1},
$$

where $n T<t<(n+1) T$. 
Proof. Using the function

$$
G[t, x(t)]=-K G[x] \sum_{k=1}^{\infty} \delta\left(\frac{t}{T}-k\right),
$$

equation (21) has the form of (18) with the Riemann-Liouville fractional derivative of order $\alpha$, where $1<\alpha \leq 2$. It allows us to use the Kilbas-Bonilla-Trujillo theorem. As a result, equation (21) with initial conditions (19) of the form (22) is equivalent to the nonlinear Volterra integral equation

$$
x(t)=\frac{c_{1}}{\Gamma(\alpha)} t^{\alpha-1}+\frac{c_{2}}{\Gamma(\alpha-1)} t^{\alpha-2}-\frac{K}{\Gamma(\alpha)} \sum_{k=1}^{\infty} \int_{0}^{t} d \tau G[x(\tau)](t-\tau)^{\alpha-1} \delta\left(\frac{\tau}{T}-k\right),
$$

where $t>0$. If $n T<t<(n+1) T$, then the integration in (25) with respect to $\tau$ gives (23).

This ends the proof.

To obtain equations of discrete map a momentum must be defined. There are two possiblities of defining the momentum:

$$
p(t)={ }_{0} D_{t}^{\alpha-1} x(t), \quad p(t)=D_{t}^{1} x(t) .
$$

Let us use the first definition. Then the momentum is defined by the fractional derivative of order $\alpha-1$. Using the definition of the Riemann-Liouville fractional derivative (17) in the form

$$
{ }_{0} D_{t}^{\alpha} x(t)=D_{t}^{2}{ }_{0} I_{t}^{2-\alpha} x(t), \quad(1<\alpha \leq 2),
$$

we define the momentum

$$
p(t)={ }_{0} D_{t}^{\alpha-1} x(t)=\frac{1}{\Gamma(2-\alpha)} \frac{d}{d t} \int_{0}^{t} \frac{x(\tau) d \tau}{(t-\tau)^{\alpha-1}}, \quad(1<\alpha \leq 2),
$$

where $x(\tau)$ is defined for $\tau \in(0, t)$. Then

$$
{ }_{0} D_{t}^{\alpha} x(t)=D_{t}^{1} p(t), \quad(1<\alpha \leq 2) .
$$

Using momentum $p(t)$ and coordinate $x(t)$, equation (21) can be represented in the Hamiltonian form

$$
{ }_{0} D_{t}^{\alpha-1} x(t)=p(t),
$$




$$
D_{t}^{1} p(t)=-K G[x(t)] \sum_{k=1}^{\infty} \delta\left(\frac{t}{T}-k\right) .
$$

Proposition 3. The Cauchy type problem for the fractional differential equations of the form (30) and (31) with the initial conditions

$$
\left({ }_{0} D_{t}^{\alpha-1} x\right)(0+)=c_{1}, \quad\left({ }_{0} D_{t}^{\alpha-2} x\right)(0+)=\left({ }_{0} I_{t}^{2-\alpha} x\right)(0+)=c_{2}
$$

is equivalent to the discrete map equations

$$
\begin{gathered}
x_{n+1}=\frac{c_{1} T^{\alpha-1}}{\Gamma(\alpha)}(n+1)^{\alpha-1}+\frac{c_{2} T^{\alpha-2}}{\Gamma(\alpha-1)}(n+1)^{\alpha-2}-\frac{K T^{\alpha}}{\Gamma(\alpha)} \sum_{k=1}^{n} G\left[x_{k}\right](n+1-k)^{\alpha-1}, \\
p_{n+1}=c_{1}-K T \sum_{k=1}^{n} G\left[x_{k}\right] .
\end{gathered}
$$

Proof. We use Proposition 2 to prove this statement. To obtain an equation for the momentum (28), we use the following fractional derivatives of power functions (see Section 2.1 in [7]),

$$
\begin{gathered}
{ }_{a} D_{t}^{\alpha}(t-a)^{\beta-1}=\frac{\Gamma(\beta)}{\Gamma(\beta-\alpha)}(t-a)^{\beta-1-\alpha}, \quad \alpha \geq 0, \quad \beta>0, \quad t>a \\
{ }_{0} D_{t}^{\alpha} t^{\alpha-k}=0, \quad k=1, \ldots, n, \quad n-1<\alpha \leq n .
\end{gathered}
$$

These equations give

$$
\begin{gathered}
{ }_{0} D_{t}^{\alpha} t^{\alpha-1}=\Gamma(\alpha), \\
{ }_{0} D_{t}^{\alpha} t^{\alpha-2}=0,
\end{gathered}
$$

and

$$
{ }_{a} D_{t}^{\alpha}(t-a)^{\alpha-1}=\Gamma(\alpha)
$$

We note that equation (23) for $x(\tau)$ can be used only if $\tau \in(n T, t)$, where $n T<t<(n+1) T$. The function $x(\tau)$ in the fractional derivative ${ }_{0} D_{t}^{\alpha}$ of the form (28) must be defined for all $\tau \in(0, t)$. We cannot take the derivative ${ }_{0} D_{t}^{\alpha}$ of the functions $(\tau-k T)^{\alpha-1}$ that are defined 
for $\tau \in(k T, t)$. In order to use equation (23) on the interval $(0, t)$, we must modify the sum in equation (23) by using the Heaviside step function. Then equation (23) has the form

$$
x(\tau)=\frac{c_{1}}{\Gamma(\alpha)} \tau^{\alpha-1}+\frac{c_{2}}{\Gamma(\alpha-1)} \tau^{\alpha-2}-\frac{K T}{\Gamma(\alpha)} \sum_{k=1}^{n} G[x(k T)](\tau-k T)^{\alpha-1} \theta(\tau-k T),
$$

where $\tau \in(0, t)$. Using the relation

$$
{ }_{0} D_{t}^{\alpha}\left((t-a)^{\alpha-1} \theta(t-a)\right)={ }_{a} D_{t}^{\alpha}(t-a)^{\alpha-1}=\Gamma(\alpha),
$$

equations (28) and (37) give

$$
p(t)=c_{1}-K T \sum_{k=1}^{n} G[x(k T)]
$$

where $n T<t<(n+1) T$. Then the solution of the left side of the $(n+1)$-th kick

$$
p_{n+1}=c_{1}-K T \sum_{k=1}^{n} G\left[x_{k}\right] .
$$

As a result, we obtain a universal map with memory in the form of equations (33) and (34).

This ends the proof.

\section{Remark 3.}

For $\alpha=n=2$ equations (33) and (34) give the usual universal map (66) and (77).

\section{Remark 4 .}

We note that the map (33) and (34) with

$$
c_{1}=p_{1}, \quad c_{2}=0
$$

was obtained in [14] in the form

$$
\begin{gathered}
x_{n+1}=\frac{T^{\alpha-1}}{\Gamma(\alpha)} \sum_{k=1}^{n} p_{k+1} V_{\alpha}(n-k+1), \\
p_{n+1}=p_{n}-K T G\left(x_{n}\right), \quad(1<\alpha \leq 2),
\end{gathered}
$$


where $p_{1}=c_{1}$, and the function $V_{\alpha}(z)$ is defined by

$$
V_{\alpha}(z)=z^{\alpha-1}-(z-1)^{\alpha-1}, \quad(z \geq 1)
$$

In Ref. [14], we obtain these map equations by using an auxiliary variable $\xi(t)$ such that

$$
{ }_{0}^{C} D_{t}^{2-\alpha} \xi(t)=x(t)
$$

The nonlinear Volterra integral equations and the general initial conditions (32) are not used in [14]. In the general case, the fractional differential equation of the kicked system (21) is equivalent to the discrete map equations

$$
\begin{gathered}
x_{n+1}=\frac{T^{\alpha-1}}{\Gamma(\alpha)} \sum_{k=1}^{n} p_{k+1} V_{\alpha}(n-k+1)+\frac{c_{2} T^{\alpha-2}}{\Gamma(\alpha-1)}(n+1)^{\alpha-2}, \\
p_{n+1}=p_{n}-K T G\left(x_{n}\right), \quad(1<\alpha \leq 2),
\end{gathered}
$$

where $p_{1}=c_{1}$. Here we take into account the initial conditions (32). The second term of the right-hand side of equation (44) is not considered in [14]. Using $-1<\alpha-2<0$, we have

$$
\lim _{n \rightarrow \infty}(n+1)^{\alpha-2}=0
$$

Therefore, the case of large values of $n$ is equivalent to $c_{2}=0$.

Let us give the proposition regarding the second definition of the momentum $p(t)=D_{t}^{1} x(t)$.

Proposition 4. The Cauchy-type problem for the fractional differential equations

$$
\begin{gathered}
D_{t}^{1} x(t)=p(t), \\
{ }_{0} D_{t}^{\alpha} x(t)=-K G[x(t)] \sum_{k=1}^{\infty} \delta\left(\frac{t}{T}-k\right), \quad(1<\alpha<2),
\end{gathered}
$$

with the initial conditions

$$
\left({ }_{0} D_{t}^{\alpha-1} x\right)(0+)=c_{1}, \quad\left({ }_{0} D_{t}^{\alpha-2} x\right)(0+)=\left({ }_{0} I_{t}^{2-\alpha} x\right)(0+)=c_{2}
$$


is equivalent to the discrete map equations

$$
\begin{gathered}
x_{n+1}=\frac{c_{1} T^{\alpha-1}}{\Gamma(\alpha)}(n+1)^{\alpha-1}+\frac{c_{2} T^{\alpha-2}}{\Gamma(\alpha-1)}(n+1)^{\alpha-2}-\frac{K T^{\alpha}}{\Gamma(\alpha)} \sum_{k=1}^{n} G\left[x_{k}\right](n+1-k)^{\alpha-1} . \\
p_{n+1}=\frac{c_{1} T^{\alpha-2}}{\Gamma(\alpha-1)}(n+1)^{\alpha-2}+\frac{c_{2}(\alpha-2) T^{\alpha-3}}{\Gamma(\alpha-1)}(n+1)^{\alpha-3}-\frac{K T^{\alpha-1}}{\Gamma(\alpha-1)} \sum_{k=1}^{n} G\left[x_{k}\right](n+1-k)^{\alpha-2} .
\end{gathered}
$$

Proof. We define the momentum

$$
p(t)=D_{t}^{1} x(t)
$$

If $n T<t<(n+1) T$, then the differentiation of (23) with respect to $t$ gives

$$
p(t)=\frac{c_{1}}{\Gamma(\alpha-1)} t^{\alpha-2}+\frac{c_{2}(\alpha-2)}{\Gamma(\alpha-1)} t^{\alpha-3}-\frac{K T}{\Gamma(\alpha-1)} \sum_{k=1}^{n} G[x(k T)](t-k T)^{\alpha-2} .
$$

Here we use the relation

$$
\Gamma(\alpha)=(\alpha-1) \Gamma(\alpha-1), \quad(1<\alpha \leq 2)
$$

Using equations (23) and (51), we can obtain the solution of the left side of the $(n+1)$-th kick (13) and (14). As a result, we have equations (49) and (50).

This ends the proof.

\section{Remark 5.}

Equations (49) and (50) describe a generalization of equations (6) and (17). If $\alpha=n=2$, and $c_{2}=x_{0}, c_{1}=p_{0}$, then equation (49) gives (6) and (77).

\section{Remark 6.}

In equations (50) and (51), we can use

$$
\frac{c_{2}(\alpha-2)}{\Gamma(\alpha-1)}=\frac{c_{2}}{\Gamma(\alpha-2)}
$$

for $1<\alpha<2$. 


\section{Remark 7.}

If we use the definition $p(t)=D_{t}^{1} x(t)$, then the Hamiltonian form of the equations of motion will be more complicated than (30) and (31) since

$$
D_{t}^{2}{ }_{0} I_{t}^{2-\alpha} x(t) \neq{ }_{0} I_{t}^{2-\alpha} D_{t}^{2} x(t)
$$

\section{Remark 8.}

Note that we use the usual momentum $p(t)=D_{t}^{1} x(t)$. In this case, the values $c_{1}$ and $c_{2}$ are not connected with $p(0)$ and $x(0)$. If we use the momentum $p(t)={ }_{0} D_{t}^{\alpha-1} x(t)$, then $c_{1}=p(0)$.

\section{Riemann-Liouville and Caputo fractional derivatives}

In Ref. [14] we consider nonlinear differential equations with Riemann-Liouville fractional derivatives. The discrete maps with memory are obtained from these equations. The problems with initial conditions for the Riemann-Liouville fractional derivative are not discussed.

The Riemann-Liouville fractional derivative has some notable disadvantages in applications in mechanics such as the hyper-singular improper integral, where the order of singularity is higher than the dimension, and nonzero of the fractional derivative of constants, which would entail that dissipation does not vanish for a system in equilibrium. The desire to use the usual

initial value problems for mechanical systems leads to the use of Caputo fractional derivatives [7, 6] rather than the Riemann-Liouville fractional derivatives.

The left-sided Caputo fractional derivative [23, 24, 25, 7] of order $\alpha>0$ is defined by

$$
{ }_{0}^{C} D_{t}^{\alpha} f(t)=\frac{1}{\Gamma(n-\alpha)} \int_{0}^{t} \frac{d \tau D_{\tau}^{n} f(\tau)}{(t-\tau)^{\alpha-n+1}}={ }_{0} I_{t}^{n-\alpha} D_{t}^{n} f(t),
$$

where $n-1<\alpha<n$, and ${ }_{0} I_{t}^{\alpha}$ is the left-sided Riemann-Liouville fractional integral of order $\alpha>0$ that is defined by

$$
{ }_{0} I_{t}^{\alpha} f(t)=\frac{1}{\Gamma(\alpha)} \int_{0}^{t} \frac{f(\tau) d \tau}{(t-\tau)^{1-\alpha}}, \quad(t>0) .
$$

This definition is, of course, more restrictive than the Riemann-Liouville fractional derivative [4, 1] in that it requires the absolute integrability of the derivative of order $n$. The Caputo 
fractional derivative first computes an ordinary derivative followed by a fractional integral to achieve the desire order of fractional derivative. The Riemann-Liouville fractional derivative is computed in the reverse order. Integration by part of (52) will lead to

$$
{ }_{0}^{C} D_{t}^{\alpha} x(t)={ }_{0} D_{t}^{\alpha} x(t)-\sum_{k=0}^{n-1} \frac{t^{k-\alpha}}{\Gamma(k-\alpha+1)} x^{(k)}(0) .
$$

It is observed that the second term in equation (54) regularizes the Caputo fractional derivative to avoid the potentially divergence from singular integration at $t=0$. In addition, the Caputo fractional differentiation of a constant results in zero

$$
{ }_{0}^{C} D_{t}^{\alpha} C=0
$$

Note that the Riemann-Liouville fractional derivative of a constant need not be zero, and we have

$$
{ }_{0} D_{t}^{\alpha} C=\frac{t^{-\alpha}}{\Gamma(1-\alpha)} C .
$$

If the Caputo fractional derivative is used instead of the Riemann-Liouville fractional derivative, then the initial conditions for fractional dynamical systems are the same as those for the usual dynamical systems. The Caputo formulation of fractional calculus can be more applicable in mechanics than the Riemann-Liouville formulation.

\section{Caputo fractional derivative and universal map with memory}

In this section, we study a generalization of differential equation (1) by the Caputo fractional derivative. The universal map with memory is derived from this fractional equation.

We consider the nonlinear differential equation of order $\alpha$, where $0 \leq n-1<\alpha \leq n$,

$$
{ }_{0}^{C} D^{\alpha} x(t)=G[t, x(t)], \quad\left(0 \leq t \leq t_{f}\right),
$$

involving the Caputo fractional derivative ${ }_{0}^{C} D_{t}^{\alpha}$ on a finite interval $\left[0, t_{f}\right]$ of the real axis, with the initial conditions

$$
\left(D_{t}^{k} x\right)(0)=c_{k}, \quad k=0, \ldots, n-1 .
$$


Kilbas and Marzan [19, 20] proved the equivalence of the Cauchy-type problem of the form (55), (56) and the Volterra integral equation of second kind

$$
x(t)=\sum_{k=0}^{n-1} \frac{c_{k}}{k !} t^{k}+\frac{1}{\Gamma(\alpha)} \int_{0}^{t} d \tau G[\tau, x(\tau)](t-\tau)^{\alpha-1}
$$

in the space $C^{n-1}\left[0, t_{f}\right]$. For $\alpha=n=2$ equation (57) gives (10).

Let us give the basic theorem regarding the nonlinear differential equation involving the Caputo fractional derivative.

Kilbas-Marzan Theorem. The Cauchy-type problem (55) and (56) and the nonlinear Volterra integral equation (57) are equivalent in the sense that, if $x(t) \in C\left[0, t_{f}\right]$ satisfies one of these relations, then it also satisfies the other.

Proof. In [19, 20] (see also [7], Theorem 3.24.) this theorem is proved by assuming that a function $G[t, x]$ for any $x \in W \subset \mathbb{R}$ belong to $C_{\gamma}\left(0, t_{f}\right)$ with $0 \leq \gamma<1, \gamma<\alpha$. Here $C_{\gamma}\left(0, t_{f}\right)$ is the weighted space of functions $f[t]$ given on $\left(0, t_{f}\right]$, such that $t^{\gamma} f[t] \in C\left(0, t_{f}\right)$. This ends the proof.

We consider the fractional differential equation of the form

$$
{ }_{0}^{C} D_{t}^{\alpha} x(t)+K G[x(t)] \sum_{k=1}^{\infty} \delta\left(\frac{t}{T}-k\right)=0, \quad(1<\alpha<2),
$$

where ${ }_{0}^{C} D_{t}^{\alpha}$ is the Caputo fractional derivative, with the initial conditions

$$
x(0)=x_{0}, \quad\left(D^{1} x\right)(0)=p_{0} .
$$

Using $p(t)=D_{t}^{1} x(t)$, equation (58) can be rewritten in the Hamilton form.

Proposition 5. The Cauchy-type problem for the fractional differential equations

$$
D_{t}^{1} x(t)=p(t)
$$




$$
{ }_{0}^{C} D_{t}^{\alpha-1} p(t)=-K G[x(t)] \sum_{k=1}^{\infty} \delta\left(\frac{t}{T}-k\right), \quad(1<\alpha<2),
$$

with the initial conditions

$$
x(0)=x_{0}, \quad p(0)=p_{0}
$$

is equivalent to the discrete map equations

$$
\begin{gathered}
x_{n+1}=x_{0}+p_{0}(n+1) T-\frac{K T^{\alpha}}{\Gamma(\alpha)} \sum_{k=1}^{n}(n+1-k)^{\alpha-1} G\left[x_{k}\right], \\
p_{n+1}=p_{0}-\frac{K T^{\alpha-1}}{\Gamma(\alpha-1)} \sum_{k=1}^{n}(n+1-k)^{\alpha-2} G\left[x_{k}\right] .
\end{gathered}
$$

Proof. We use the Kilbas-Marzan theorem with the function

$$
G[t, x(t)]=-K G[x(t)] \sum_{k=1}^{\infty} \delta\left(\frac{t}{T}-k\right) .
$$

The Cauchy-type problem (58) and (59) is equivalent to the Volterra integral equation of second kind

$$
x(t)=x_{0}+p_{0} t-\frac{K}{\Gamma(\alpha)} \sum_{k=1}^{\infty} \int_{0}^{t} d \tau(t-\tau)^{\alpha-1} G[x(\tau)] \delta\left(\frac{t}{T}-k\right),
$$

in the space of continuously differentiable functions $x(t) \in C^{1}\left[0, t_{f}\right]$.

If $n T<t<(n+1) T$, then equation (65) gives

$$
x(t)=x_{0}+p_{0} t-\frac{K T}{\Gamma(\alpha)} \sum_{k=1}^{n}(t-k T)^{\alpha-1} G[x(k T)] .
$$

We define the momenta

$$
p(t)=D_{t}^{1} x(t)
$$

Then equations (66) and (67) give

$$
p(t)=p_{0}-\frac{K T}{\Gamma(\alpha-1)} \sum_{k=1}^{n}(t-k T)^{\alpha-2} G[x(k T)], \quad(n T<t<(n+1) T),
$$

where we use $\Gamma(\alpha)=(\alpha-1) \Gamma(\alpha-1)$. 
The solution of the left side of the $(n+1)$-th kick (13) and (14) can be represented by equations (63) and (64), where we use the condition of continuity $x\left(t_{n}+0\right)=x\left(t_{n}-0\right)$.

This ends the proof.

\section{Remark 9.}

Equations (63) and (64) define a generalization of the universal map. This map is derived from a fractional differential equation with Caputo derivatives without any approximations. The main property of the suggested map is a long-term memory that means that their present state depends on all past states with a power-law form of weights.

\section{Remark 10.}

If $\alpha=2$, then equations ([63) and (64) give the universal map of the form (6) and (17) that is equivalent to equations (2). As a result, the usual universal map is a special case of this universal map with memory.

\section{Remark 11.}

By analogy with Proposition 5, it is easy to obtain the universal map with memory from fractional equation (58) with $\alpha>2$.

\section{Conclusion}

The suggested discrete maps with memory are generalizations of the universal map. These maps describe fractional dynamics of complex physical systems. The suggested universal maps with memory are equivalent to the correspondent fractional kicked differential equations. We obtain a discrete map from fractional differential equation by using the equivalence of the Cauchy-type problem and the nonlinear Volterra integral equation of second kind. An approximation for fractional derivatives of these equations is not used.

It is important to obtain and to study discrete maps which correspond to the real physical systems described by the fractional differential equations. Media with memory in mechanics and electrodynamics, we can consider viscoelastic and dielectric materials as a media with memory. We note that the dielectric susceptibility of a wide class of dielectric materials follows, over 
extended frequency ranges, a fractional power-law frequency dependence that is called the "universal" response [15, 16]. As was proved in [17, 18], the electromagnetic fields in such dielectric media are described by differential equations with fractional time derivatives. These fractional equations for electromagnetic waves in dielectric media are common to a wide class of materials, regardless of the type of physical structure, chemical composition, or of the nature of the polarizing species, whether dipoles, electrons or ions. We hope that it is possible to obtain the discrere maps with memory which correspond to the real dielectric media described by the fractional differential equations.

\section{Acknowledgments}

This work was supported by the Office of Naval Research, Grant No. N00014-02-1-0056 and Rosnauka No.02.740.11.0244.

\section{References}

[1] B.V. Chirikov, "A universal instability of many dimensional oscillator systems" Physics Reports 52(5), (1979) 263-379.

[2] R.Z. Sagdeev, D.A. Usikov, G.M. Zaslavsky, Nonlinear Physics: From the Pendulum to Turbulence and Chaos (Harwood Academic Publishers, New-York, 1988).

[3] G.M. Zaslavsky, Hamiltonian Chaos and Fractional Dynamics (Oxford University Press, Oxford, 2005).

[4] S.G. Samko, A.A. Kilbas, O.I. Marichev, Fractional Integrals and Derivatives Theory and Applications (Gordon and Breach, New York, 1993).

[5] K. Miller, B. Ross, An Introduction to the Fractional Calculus and Fractional Differential Equations (Wiley, New York, 1993).

[6] I. Podlubny, Fractional Differential Equations (Academic Press, San Diego, 1999).

[7] A.A. Kilbas, H.M. Srivastava, J.J. Trujillo, Theory and Application of Fractional Differential Equations (Elsevier, Amsterdam, 2006). 
[8] A. Fulinski, A.S. Kleczkowski, "Nonlinear maps with memory" Physica Scripta 35(2), (1987) 119-122.

[9] E. Fick, M. Fick, G. Hausmann, "Logistic equation with memory" Physical Review A 44(4), (1991) 2469-2473.

[10] K. Hartwich, E. Fick, "Hopf bifurcations in the logistic map with oscillating memory" Physics Letters A 177(4/5), (1993) 305-310.

[11] M. Giona, "Dynamics and relaxation properties of complex systems with memory" Nonlinearity 4(3), (1991) 991-925.

[12] J.A.C. Gallas, "Simulating memory effects with discrete dynamical systems" Physica A 195(3/4), (1993) 417-430; "Erratum" Physica A 198(1/2), (1993) 339-339.

[13] A.A. Stanislavsky, "Long-term memory contribution as applied to the motion of discrete dynamical system" Chaos 16(4), (2006) 043105.

[14] V.E. Tarasov, G.M. Zaslavsky, "Fractional equations of kicked systems and discrete maps" Journal of Physics A 41(43), (2008) 435101. (arXiv:1107.3953)

[15] A.K. Jonscher, Universal Relaxation Law, (Chelsea Dielectrics Press, London, 1996).

[16] A.K. Jonscher, "Dielectric relaxation in solids" J. Physics D Appl. Phys. 32 (1999) R57R70.

[17] V.E. Tarasov, "Universal electromagnetic waves in dielectric" Journal of Physics: Condensed Matter. Vol.20. No.17. (2008) 175223. (arXiv:0907.2163)

[18] V.E. Tarasov, "Fractional equations of Curie-von Schweidler and Gauss laws" Journal of Physics: Condensed Matter. Vol.20. No.14. (2008) 145212. (arXiv:0907.1837)

[19] A.A. Kilbas, S.A. Marzan, "The Cauchy problem for differential equations with fractional Caputo derivative" Doklady Mathematics 70(3), (2004) 841-845; Translated from Doklady Akademii Nauk 399(1), (2004) 7-11. (in Russian).

[20] A.A. Kilbas, S.A. Marzan, "Nonlinear differential equations with the Caputo fractional derivative in the space of continuously differentiable functions" Differential Equations 41(1), (2005) 84-89; Translated from Differentsialnye Uravneniya 41(1), (2005) 82-86. (in Russian). 
[21] A.A. Kilbas, B. Bonilla, J.J. Trujillo, "Nonlinear differential equations of fractional order is space of integrable functions" Doklady Mathematics 62(2), (2000) 222-226; Translated from Doklady Akademii Nauk 374(4), (2000) 445-449. (in Russian).

[22] A.A. Kilbas, B. Bonilla, J.J. Trujillo, "Existence and uniqueness theorems for nonlinear fractional differential equations" Demonstratio Mathematica 33(3), (2000) 583-602.

[23] M. Caputo, "Linear models of dissipation whose Q is almost frequency independent. Part II" Geophysical Journal of the Royal Astronomical Society 13(5), (1967) 529-539.

[24] M. Caputo, F. Mainardi, "A new dissipation model based on memory mechanism" Pure and Applied Geophysics 91(1), (1971) 134-147.

[25] A. Carpinteri, F. Mainardi (Eds), Fractals and Fractional Calculus in Continuum Mechanics (Springer, Wien, 1997).

[26] V.E. Tarasov, Fractional Dynamics: Application of Fractional Calculus to Dynamics of Particles, Fields and Media (Springer, HEP, 2011) 504 pages. 